\title{
Pharmacokinetic modeling of intravenous sildenafil in newborns with congenital diaphragmatic hernia
}

\author{
Suzan C. M. Cochius - den Otter $^{1}$ (D) Florian Kipfmueller $^{2} \cdot$ Brenda C. M. de Winter $^{3} \cdot$ Karel Allegaert $^{1,4} \cdot$ Dick Tibboel $^{1}$. \\ Andreas Mueller ${ }^{2} \cdot$ Birgit C. P. Koch ${ }^{3}$
}

Received: 30 May 2019 / Accepted: 15 September 2019/Published online: 18 November 2019

(C) The Author(s) 2019

\begin{abstract}
Purpose We developed a pharmacokinetic model of intravenous sildenafil in newborns with congenital diaphragmatic hernia $(\mathrm{CDH})$ to achieve a target plasma concentration of over $50 \mu \mathrm{g} / \mathrm{l}$.

Methods Twenty-three CDH newborns with pulmonary hypertension (64 blood samples) received intravenous sildenafil. Patients received a loading dose of $0.35 \mathrm{mg} / \mathrm{kg}$ (IQR $0.16 \mathrm{mg} / \mathrm{kg}$ ) for $3 \mathrm{~h}$, followed by a continuous infusion of $1.5 \mathrm{mg} / \mathrm{kg} /$ day (IQR $0.1 \mathrm{mg} / \mathrm{kg} / \mathrm{day}$ ). For model development, non-linear mixed modeling was used. Inter-individual variability (IIV) and inter-occasion variability were tested. Demographic and laboratory parameters were evaluated as covariates. Normalized prediction distribution errors (NPDE) and visual predictive check (VPC) were used for model validation.

Results A two-compartment disposition model of sildenafil and a one-compartment disposition model of desmethyl sildenafil (DMS) was observed with IIV in sildenafil and DMS clearance and volume of distribution of sildenafil. NPDE and VPC revealed adequate predictability. Only postnatal age increased sildenafil clearance. This was partly compensated by a higher DMS concentration, which also has a therapeutic effect. In this small group of patients, sildenafil was tolerated well.

Conclusions This model for sildenafil in $\mathrm{CDH}$ patients shows that concentration-targeted sildenafil dosing of $0.4 \mathrm{mg} / \mathrm{kg}$ in $3 \mathrm{~h}$, followed by $1.6 \mathrm{mg} / \mathrm{kg} /$ day continuous infusion achieves appropriate sildenafil plasma levels.
\end{abstract}

Keywords Sildenafil $\cdot$ Pharmacokinetics $\cdot$ Modeling $\cdot$ Congenital diaphragmatic hernia $\cdot$ Cardiovascular tolerance

What is already known about this subject:

- Therapy resistant pulmonary hypertension $(\mathrm{PH})$ in congenital diaphragmatic hernia $(\mathrm{CDH})$ patients has a high mortality rate.

- Sildenafil is used for the treatment of PH, but data on optimal dosing and pharmacokinetics (PK) are lacking.

- In this study, we developed a PK model for sildenafil in $\mathrm{CDH}$ patients. What this study adds:

- An intravenous sildenafil loading dose of $0.4 \mathrm{mg} / \mathrm{kg}$ in $3 \mathrm{~h}$ followed by continuous infusion of $1.6 \mathrm{mg} / \mathrm{kg}$ /day achieves therapeutic sildenafil plasma levels in $\mathrm{CDH}$ patients in approximately $1 \mathrm{~h}$.

- The current PK model is the first step toward concentration range targeted sildenafil dosing in $\mathrm{CDH}$ patients.

Electronic supplementary material The online version of this article (https://doi.org/10.1007/s00228-019-02767-1) contains supplementary material, which is available to authorized users.

Suzan C. M. Cochius - den Otter

s.denotter@erasmusmc.nl

1 Department of Pediatrics, Intensive Care and Pediatric Surgery, Erasmus MC University Medical Center, Wytemaweg 80, 3015, CN Rotterdam, the Netherlands
2 Department of Neonatology and Pediatric Critical Care Medicine, University Children's Hospital, Bonn, Germany

3 Department of Pharmacy, Erasmus MC University Medical Center, Rotterdam, The Netherlands

4 Department of Development and Regeneration, KU, Leuven, Belgium 


\section{Introduction}

Congenital diaphragmatic hernia $(\mathrm{CDH})$ is a developmental defect of the diaphragm with abnormal lung development and pulmonary vasculature growth, resulting in pulmonary hypertension $(\mathrm{PH})$ [1]. $\mathrm{CDH}$ is associated with a reported mortality of approximately $27 \%$ in live-born patients since the implementation of international treatment guidelines [2-4]. PH is one of the most important risk factors for poor outcome in infants with $\mathrm{CDH}[1,5,6]$.

During fetal life, high resistance in the pulmonary circulation is normal and causes most of the blood flow to bypass the lungs through the ductus arteriosus. As part of normal transition, the pulmonary vascular resistance drops immediately after birth and the blood flow through the lung vasculature increases significantly. Normal values of pulmonary vascular pressures, similar to healthy adults, are usually reached around the age of 2 months [7]. In infants with $\mathrm{CDH}$, the pulmonary vascular resistance often does not drop adequately, due to increased vascular reactivity, excessive muscularization of the pulmonary arterioles, and increased thickness of the arterial media and adventitia [8].

Intravenous sildenafil, a phosphodiesterase 5 (PDE5) inhibitor that increases cyclic guanosine monophosphate (cGMP) resulting in pulmonary vasodilation, is increasingly used in infants with $\mathrm{CDH}$, with promising results [9-11]. In newborns with persistent pulmonary hypertension of the newborn (PPHN) without $\mathrm{CDH}$, sildenafil improves oxygenation index (OI) and survival [12]. However, data on optimal dosing, pharmacokinetics (PK), and dynamics are scarce in newborns, and CDH patients are often excluded from trials. PK of intravenous sildenafil in term neonates with PPHN, using a two-compartment model, shows a threefold increase in sildenafil clearance and its active metabolite, desmethyl sildenafil (DMS), in the first week of life [13]. DMS has a 50\% potency compared to sildenafil [14]. Volume of distribution was fourfold higher than in adults, resulting in a longer half-life [13].

Also, long-term safety data are scarce [15]. In a study evaluating safety using a dose-escalating strategy, intravenous sildenafil was well tolerated and it improved oxygenation when using a higher infusion dose. With a 3-h loading dose of 0.4 $\mathrm{mg} / \mathrm{kg}$ and a continuous infusion of $1.6 \mathrm{mg} / \mathrm{kg} /$ day, target plasma concentration was achieved without causing hypotension [16].

As $\mathrm{PH}$ is a major determent of survival in patients with $\mathrm{CDH}$, data on the PK and pharmacodynamics of sildenafil are urgently needed. The objective of this study was to develop a PK model and dosing regimen for sildenafil in $\mathrm{CDH}$ patients.

\section{Methods}

\section{Study design}

This open-label study was conducted in two level-3 referral centers for $\mathrm{CDH}$ in Germany and the Netherlands between November 2013 and September 2015. Local ethical review boards approved the protocol. Twenty-three newborns with $\mathrm{CDH}$ and clinical signs of $\mathrm{PH}$ were treated with intravenous sildenafil. Patients received a loading dose of $0.35 \mathrm{mg} / \mathrm{kg}$ (IQR $0.16 \mathrm{mg} / \mathrm{kg}$ ) for $3 \mathrm{~h}$, followed by a continuous infusion of $1.5 \mathrm{mg} / \mathrm{kg} /$ day (IQR $0.1 \mathrm{mg} / \mathrm{kg} /$ day). However, many different regimens were being used; ten patients received a loading dose of $0.4 \mathrm{mg} / \mathrm{kg}$ in $3 \mathrm{~h}$, followed by continuous infusion of $1.6 \mathrm{mg} / \mathrm{kg} / \mathrm{day}$; in one patient, the infusion was very slowly increased over time, starting with $0.2 \mathrm{mg} / \mathrm{kg} / \mathrm{day}$, in others no loading dose was given and a continuous infusion was started, ranging from 1.4 to $4 \mathrm{mg} / \mathrm{kg} /$ day. Target plasma sildenafil levels over $50 \mu \mathrm{g} / \mathrm{l}$ were aimed for, assuming that the same target range is applicable in $\mathrm{CDH}$ patients compared to other causes of neonatal PH [16]. DMS was not added to calculate this target range because the target range for DMS is unknown and DMS was also not taken into account in the study of Steinhorn et al. [16]. The patients were treated as per protocol according to international consensus [3]. Patient and baseline characteristics of the 23 patients are provided in Supplementary Table 1. All patients received inotropic drug support and were treated with inhaled nitric oxide (iNO). One patient started treatment with bosentan in the second week of life. Thirteen patients needed extracorporeal membrane oxygenation (ECMO) (median time of start 8:15 h after birth, IQR 9:39 h), of whom two were already on ECMO before sildenafil initiation, overall mortality was $26 \%$.

Primary outcomes were the PK of sildenafil and its active metabolite, DMS. Safety data were collected as secondary outcome variables. Oxygenation index (OI) was used to evaluate the clinical effect of sildenafil on PH. Hypotension was defined as mean blood pressure lower than gestational age in weeks. The Vasoactive inotropic score (VIS) was used to evaluate cardiovascular tolerance. This is a scoring system used for the amount of inotropic support needed and is negatively associated with long-term outcome [17]. OI and VIS were analyzed using linear regression analysis.

\section{Laboratory analysis}

We used $50 \mu \mathrm{l}$ EDTA blood and $200 \mu \mathrm{l}$ internal standard solution (vardenafil in methanol) was added. This solution was vortexed for $10 \mathrm{~s}$ and centrifuged for $5 \mathrm{~min}$. A supernatant of $200 \mu \mathrm{l}$ was added to the insert vial, which was used in the 
Thermo TQS Vantage LC-MS/MS. Column $2.1 \times 100 \mathrm{~mm}$ Waters Acquity CSH C18 1.7 um. The mobile phase A consisted of $2 \mathrm{mM}$ ammonium acetate in $0.1 \%$ formic acid in water. The mobile phase $\mathrm{B}$ consisted of $2 \mathrm{mM}$ ammonium acetate in $0.1 \%$ formic acid in LC-MS methanol. Flow rate was $0.5 \mathrm{ml} / \mathrm{min}$. The mobile phase composition changed linearly during analysis in a percentage mobile phase A (from 80 to $0 \%$ ) and B (from 2 to $100 \%$ ). Total analysis time was 4 min. The injected volume was $10 \mu \mathrm{l}$. The method was validated according to FDA guidelines between $2-1000 \mu \mathrm{g} / 1$ for sildenafil and 2-500 $\mu \mathrm{g} / \mathrm{l}$ for DMS [18].

\section{Population PK modeling}

PK analysis was conducted with non-linear mixed-effects modeling using NONMEM®) version 7.2 (ICON Development Solutions, Ellicott City, MD) and PsN® (version 3.7.6). Pirana software was used as an interface between NONMEM, R (version 3.2.2) and Xpose (version 4). Using NONMEM, we could estimate average PK parameters for the population as well as inter-individual variability (IIV), interoccasion variability (IOV), and residual error.

\section{Base model development}

One- two- and three-compartment models were tested for sildenafil and DSM, using the first-order conditional estimation method with interaction (FOCE+I). First, a structural model for sildenafil was developed. IIV and IOV, with occasion defined per day, were assessed on each parameter using an exponential model. Residual variability was first tested with an additive and proportional error for each component. The parameters for the base model for sildenafil were fixed when the model for the metabolite was developed. In the combined model, we estimated all parameters.

Model selection was based on minimum objective function values, parameter precision, error estimates, shrinkage values, and visual inspection of the goodness of fit plots. Shrinkage was calculated for all model parameters. A shrinkage value below $20 \%$ was considered acceptable [19].

\section{Covariate model development}

Demographic and laboratory characteristics including postnatal age, gender, creatinine, urea, aspartate transaminase (ASAT), and alanine transaminase (ALAT) were evaluated as potential model covariates. Allometric scaling was used to account for variability in PK parameters owing to differences in bodyweight [20]. Covariates that significantly improved the model in univariate analysis, defined as $p \leq 0.05$, were added to the full model. A backward elimination process was subsequently performed with statistical significance indicated by $p \leq 0.001$. Continuous covariates were normalized to the population median values and incorporated as power model functions (Eq. 1). Categorical covariates were transformed to binary covariates and incorporated as shown in Eq. 2.

$\theta_{\mathrm{i}}=\theta_{\text {pop }} *\left(\frac{\operatorname{cov}_{\mathrm{i}}}{\operatorname{cov}_{\mathrm{m}}}\right)^{\theta \operatorname{cov}}$

$\theta_{\mathrm{i}}=\theta_{\text {pop }} * \theta_{\text {cov }}{ }^{\operatorname{cov}_{i}}$

With $\theta \mathrm{i}$ being the individual model predicted $\mathrm{PK}$ parameter (e.g., clearance) for an individual with covariate value $\operatorname{cov}_{i}$, $\theta$ pop being the population estimate for that parameter, $\operatorname{cov}_{\mathrm{m}}$ representing the median covariate value and $\theta \operatorname{cov}$ the covariate effect. In the equation for categorical covariates, $\operatorname{cov}_{i}$ is either 1 or 0 .

\section{Model evaluation}

We used multiple procedures to assess the robustness of the parameter estimates and to validate the final model. First of all, a bootstrap resampling method was applied [21]. Thousand bootstrap datasets were generated by sampling randomly from the original dataset with replacement. The validity of the model was evaluated by comparing the median values and their corresponding $95 \%$ confidence intervals of the bootstrap replicates with the estimates of the original dataset.

Subsequently, the model was validated using both visual predictive check (VPC) by simulating 500 datasets and a normalized prediction distribution errors (NPDE) analysis [22, 23]. The VPCs were prediction corrected and stratified for the covariates that are included in the final model. NPDE is a simulation-based diagnostic that can be used to evaluate models developed on datasets with variable dosing regimens. The analytical value of this method has been previously described by Comets et al. [23].

\section{Concentration effect relationship}

Drug concentrations were simulated on the time points of blood pressure measurement to find a relationship between the concentration of sildenafil, DMS, and hypotension (mean blood pressure lower than gestational age), assuming a $50 \%$ activity of DMS compared with sildenafil. The relationship was tested using Mann-Whitney statistical testing. 


\section{Results}

Sixty-four samples were taken at different time points between 1 and $385 \mathrm{~h}$ after the start of the infusion, 34 of these samples were taken during ECMO. None of the patients received hemofiltration.

Median sildenafil level of all patients was 200 (range 42262) $\mu \mathrm{g} / \mathrm{l}$ at $3 \mathrm{~h}$ and increased to $366(19-506) \mu \mathrm{g} / \mathrm{l}$ at $12 \mathrm{~h}$. Infants receiving a loading dose of $0.4 \mathrm{mg} / \mathrm{kg}$ in $3 \mathrm{~h}$ achieved sildenafil levels ranging between 190 and $262 \mu \mathrm{g} / \mathrm{l}$ at $3 \mathrm{~h}$ and between 346 and $506 \mu \mathrm{g} / \mathrm{l}$ after $12 \mathrm{~h}$.

Median DMS level within the first $12 \mathrm{~h}$ was 20 (range 985) $\mu \mathrm{g} / \mathrm{l}$ with a slow increase to a median of 65 (range 17-92) $\mu \mathrm{g} / \mathrm{l}$ between 24 and $48 \mathrm{~h}$.

To evaluate the effect of sildenafil on OI and VIS, numbers were too small. However, in only one patient, sildenafil infusion was temporarily stopped after $1 \mathrm{~h}$ due to hypotension that could not be sufficiently treated with inotropes. One patient had two episodes of hypotension, after $7 \mathrm{~h}$ and 7 days, for which the sildenafil was decreased for the same reason. This patient also developed pulmonary hemorrhage after 6 days of sildenafil infusion. In both patients, no correlation with high sildenafil levels was seen. No other adverse events were seen.

\section{Base model and covariate analysis}

The model included two-compartment disposition of sildenafil and one-compartment disposition of DMS with IIV in both clearance of sildenafil and DMS, and in volume of distribution of sildenafil. The residual error was described with a proportional error model. Allometric scaling with fixed exponents ( $0.75 \mathrm{CL}$ and $\mathrm{Q}$ and 1 for $\mathrm{Vd})$ improved the model. Estimation of the exponent did not result in further improvement.

The base two-compartment model with allometric scaling was used as reference for the covariate analysis. After graphical analysis, the univariate analysis resulted in the following covariates: ECMO, postnatal age, urea, ALAT, and weight.
With a median age of 2.4 days, postnatal age was the only significant covariate after backward elimination. Increase in age resulted in increased sildenafil clearance, as can be seen in Fig. 1. Age is presented in 3 groups: 2, 6 and 10 days. When age increases, the clearance highly increases. If age is increased from 2.4 to 10 days, clearance is increased with a factor of 4 (Table 1). Shrinkage (residual error in the model) is good with regards to clearance. Shrinkage is high for distribution volume, resulting in a large residual error.

\section{Evaluation of the final model}

All estimates were within the limits, given the criteria as defined. Goodness-of-fit plots of the final model showed that the population predictions and individual predictions were evenly distributed around the line of unity when compared with observed concentrations, and the conditional weighted residuals were normally distributed over time (Fig. 2). A bootstrap analysis with 1000 bootstrap replicates was performed to obtain 95\% confidence intervals for all PK parameters.

Owing to minimization and boundary errors, the bootstrap results were recomputed without filtering the samples (1000 runs computed: 495 runs successful). Results of the bootstrap are shown in Table 1.

In the VPC, a large variation was present. However, the median and the variability were within the corresponding simulations (Fig. 3). This demonstrates the good predictive performance of the final model in the internal validation. Evaluation of the predictive performance with NPDE analysis showed adequate predictive ability, with distribution of the NPDEs not significantly deviating from a normal distribution (Figure 4 in the supplement).

\section{Concentration effect relationship}

To investigate the relationship between blood pressure and drug concentration, simulations of drug concentrations were
Fig. 1 Simulation of the current dose in which $0.4 \mathrm{mg} / \mathrm{kg}$ was given in $3 \mathrm{~h}$, followed by 1.6 $\mathrm{mg} / \mathrm{kg} /$ day. This leads to concentrations within the therapeutic range. However, in the group with the youngest age ( 2 days), the concentration is in higher target range. The therapeutic range of 50-300 ug/l is marked in yellow

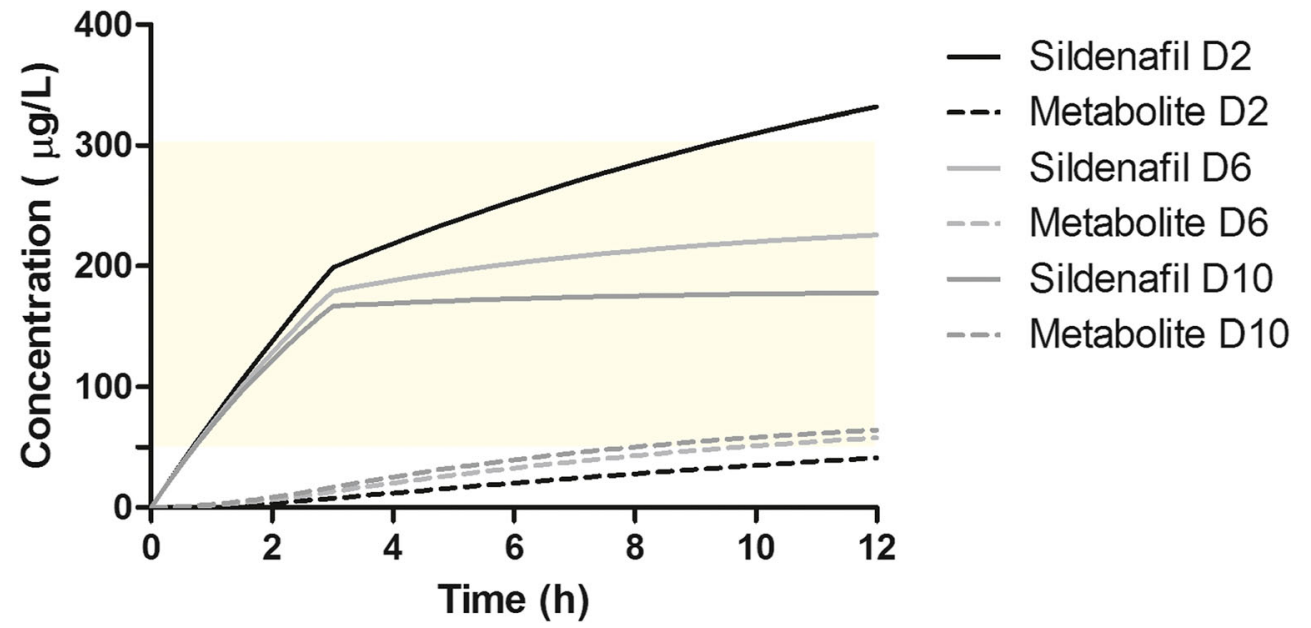


Table 1 Parameter estimates of the base model, final model, and bootstrap analysis

\begin{tabular}{llll}
\hline & Base model & Final model (rse I \%) & $\begin{array}{l}\text { Bootstrap results } \\
\text { Median [95\% CI] }\end{array}$ \\
\hline OFV & 1191 & 1160 & 1154 [918-1372] \\
CL sildenafil (L/h) & 6.7 & $5.2(19)$ & $5.1[3.7-7.0]$ \\
Age*(days) & & $0.58(26)$ & $0.58[0.3-0.9]$ \\
V1 sildenafil (L) & 75.1 & $115(30)$ & $118[61-159]$ \\
V2 sildenafil (L) & 10 & $10(\mathrm{na})$ & NA \\
Q sildenafil (L/h) & 44.3 & $221(3)$ & $330[1.9-13719]$ \\
Cl metabolite (L/h) & 24.2 & $25.9(12)$ & $26.6[21.2-32.6]$ \\
V3 metabolite & 1040 & $366(83 \%)$ & $287[79-999]$ \\
IIV (\%) & & & $24[5-65]$ \\
Cl sildenafil & 50.3 & $51.3(24 \%)$ & $5[0-35]$ \\
V1 sildenafil & 32.2 & $21.0(55 \%)$ & $15[2-51]$ \\
Cl metabolite & 37.8 & $40.5(25 \%)$ & $0.5[0.4-0.6]$ \\
Residual variability & & & 0.4 [0.4-0.5] \\
Proportional Cl sildenafil & 0.60 & $0.50(10)$ &
\end{tabular}

$* \mathrm{Cl}=\mathrm{Cl}$ (sildenafil)*(weight in $\mathrm{kg} / 70) * * 0.75 * \mathrm{EXP}(\mathrm{IIV}$ sildenafil $) *(\mathrm{AGE} / 2.4) * * 0.58$,

Shrinkage: Base model Cl sildenafil 12\%, Cl metabolite 26\%, V1 61\%; final model Cl sildenafil 11\%, V1 59\%, and $\mathrm{Cl}$ metabolite $19 \%$

performed on the time points of blood pressure measurement. No correlation was seen.

\section{Discussion}

Although intravenous sildenafil is increasingly used in $\mathrm{CDH}$ patients, a structured analysis of PK data was lacking. Therefore, the objective of the study was to develop a PK model and dosing regimen for intravenous sildenafil in newborns with $\mathrm{CDH}$. A loading dose of $0.4 \mathrm{mg} / \mathrm{kg}$ in $3 \mathrm{~h}$ followed by continuous infusion of $1.6 \mathrm{mg} / \mathrm{kg} /$ day achieves target sildenafil plasma levels within $3 \mathrm{~h}$.

Only 5 RCTs with a total of 166 patients have evaluated the effect of sildenafil in newborns, all excluding patients with congenital anomalies, including $\mathrm{CDH}$. When sildenafil was compared with placebo or $\mathrm{MgSO} 4$, a decrease in OI and mortality was seen. When sildenafil was added to inhaled NO, there was no difference in outcome [24-28]. In CDH patients, only retrospective data are available. A decrease in pulmonary vascular resistance index and an increase in cardiac output were found in a small group of oral sildenafil-treated infants with $\mathrm{CDH}$ refractory to iNO [9]. However, there is a large interpatient variability in pharmacokinetics of oral sildenafil [29]. Intravenous sildenafil in $\mathrm{CDH}$ patients was associated with improved OI and the reversal of the right-to-left shunt ratio over the ductus arteriosus. However, a significant increase in vasopressor support was also observed [10, 30].

We found that postnatal age increases sildenafil clearance suggesting maturation of the expression of hepatic CYP enzymes involved, as also observed by Mukherjee et al. Sildenafil metabolism is mostly mediated by two enzymes; CYP3A4 and CYP2C9 [31]. The activity of both enzymes is very low at birth and increases substantially during the first weeks of life [32-34]. Also, the improvement of the clinical condition possibly increases the metabolic activity of the liver $[32,35,36]$. The increase in clearance shows a non-linear increase over time and is stronger in younger age as commonly seen in infants $[13,35]$. This increase in clearance lowers sildenafil plasma levels which are partly compensated with the increase of DMS concentrations in time (Figures 1 and 5 in the supplement).

A loading dose of $0.4 \mathrm{mg} / \mathrm{kg}$ in $3 \mathrm{~h}$ followed by continuous infusion achieved target sildenafil plasma levels within $3 \mathrm{~h}$ (Fig. 1). This dosing regime was first described by Steinhorn et al. in a dose-escalating trial in newborns with PPHN in the absence of $\mathrm{CDH}$. Is this small group of 4 patients, sildenafil plasma levels reached $107.78 \mu \mathrm{g} / \mathrm{l}$ at $3 \mathrm{~h}$ and $246.28 \mu \mathrm{g} / \mathrm{l}$ at 24 $\mathrm{h}$, and it was well tolerated and improved the OI [16]. Our data suggest that there is no major difference in the PK of sildenafil in $\mathrm{CDH}$ patients, although our patients reached therapeutic plasma levels earlier, after approximately $1 \mathrm{~h}$. As the clinical condition of $\mathrm{CDH}$ patients can deteriorate quickly, the ideal dosing regimen reaches therapeutic plasma levels as soon as possible with the least possible side effects. This seems feasible with the dosing regimen we propose. Evaluation of the clinical effects of sildenafil and DMS in CDH patients suggested relative cardiovascular tolerance but a more clear effect could not be seen. With the amount of patients and samples, we could develop a PK model, however, for the secondary 

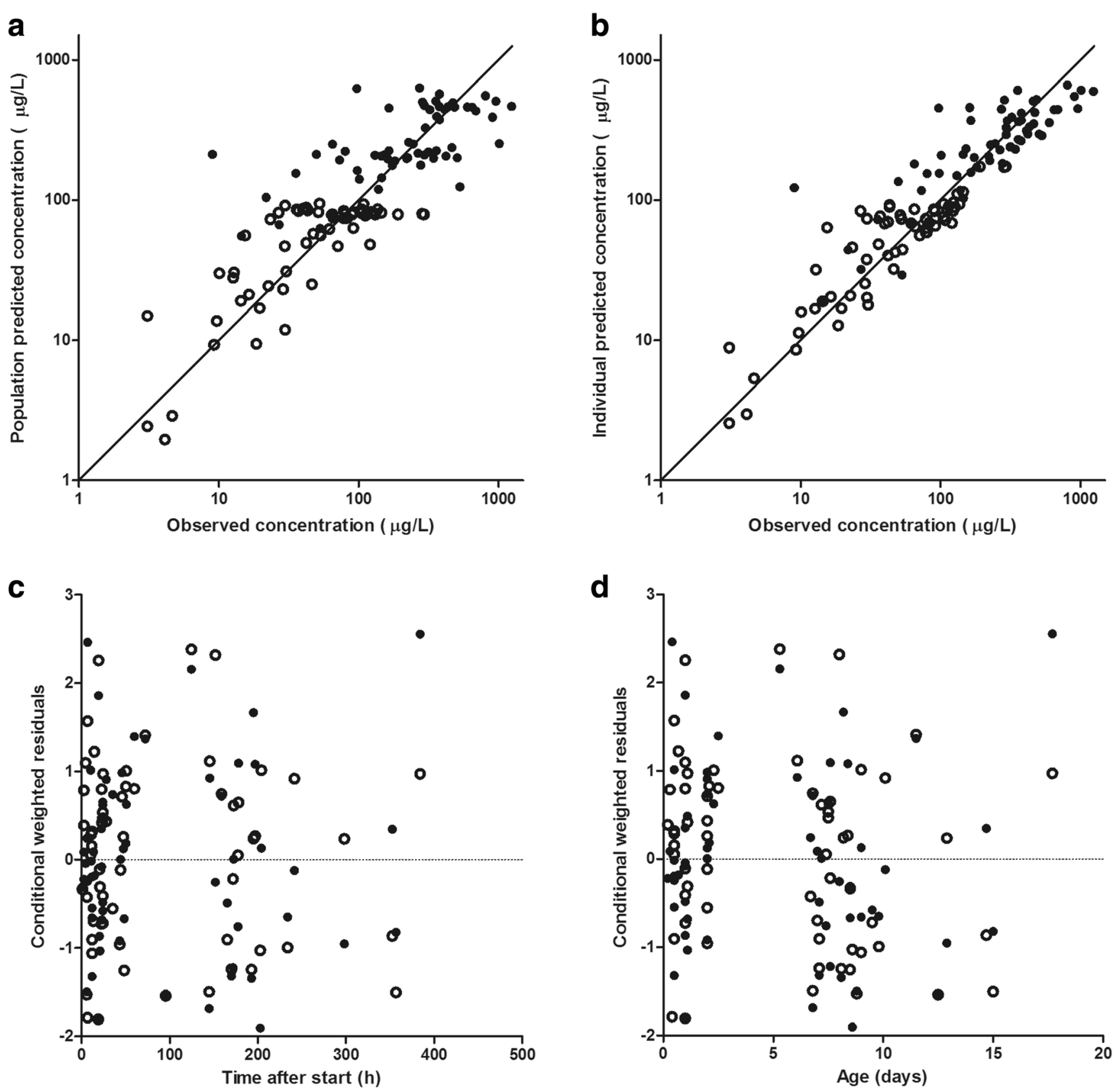

Fig. 2 Goodness of fit plots. Goodness-of-fit plots of the final model. a Observed concentration (DV) plotted against predicted concentration (PRED). b DV plotted against individual predicted concentration

(IPRED). c Conditional weighted residuals versus time after start. d Conditional weighted residuals versus age

There are some limitations of the study. As $\mathrm{CDH}$ is a outcome, parameter numbers were too low. Also, $57 \%$ of patients needed ECMO treatment, at that point making the OI invalid to analyze the effect of sildenafil on PH. As these are retrospective data, echocardiography was not performed at predefined time points, making it impossible to evaluate the effect of sildenafil on $\mathrm{PH}$ in these patients. However, in this selected group of critically ill $\mathrm{CDH}$ patients, $\mathrm{PH}$ mortality was $26 \%$, whereas reported overall mortality for $\mathrm{CDH}$ patients is $27 \%$. rare disease, 23 patients is a relatively large group, but to test the model and to better achieve patient targeted dosing, prospective external validation is needed. A multicenter RCT, the CoDiNOS trial, has started in Europe, comparing iNO with intravenous sildenafil as first-line treatment of PH in CDH patients (NTR6982). In this trial, sildenafil and DMS plasma levels will be collected to externally validate the model. 

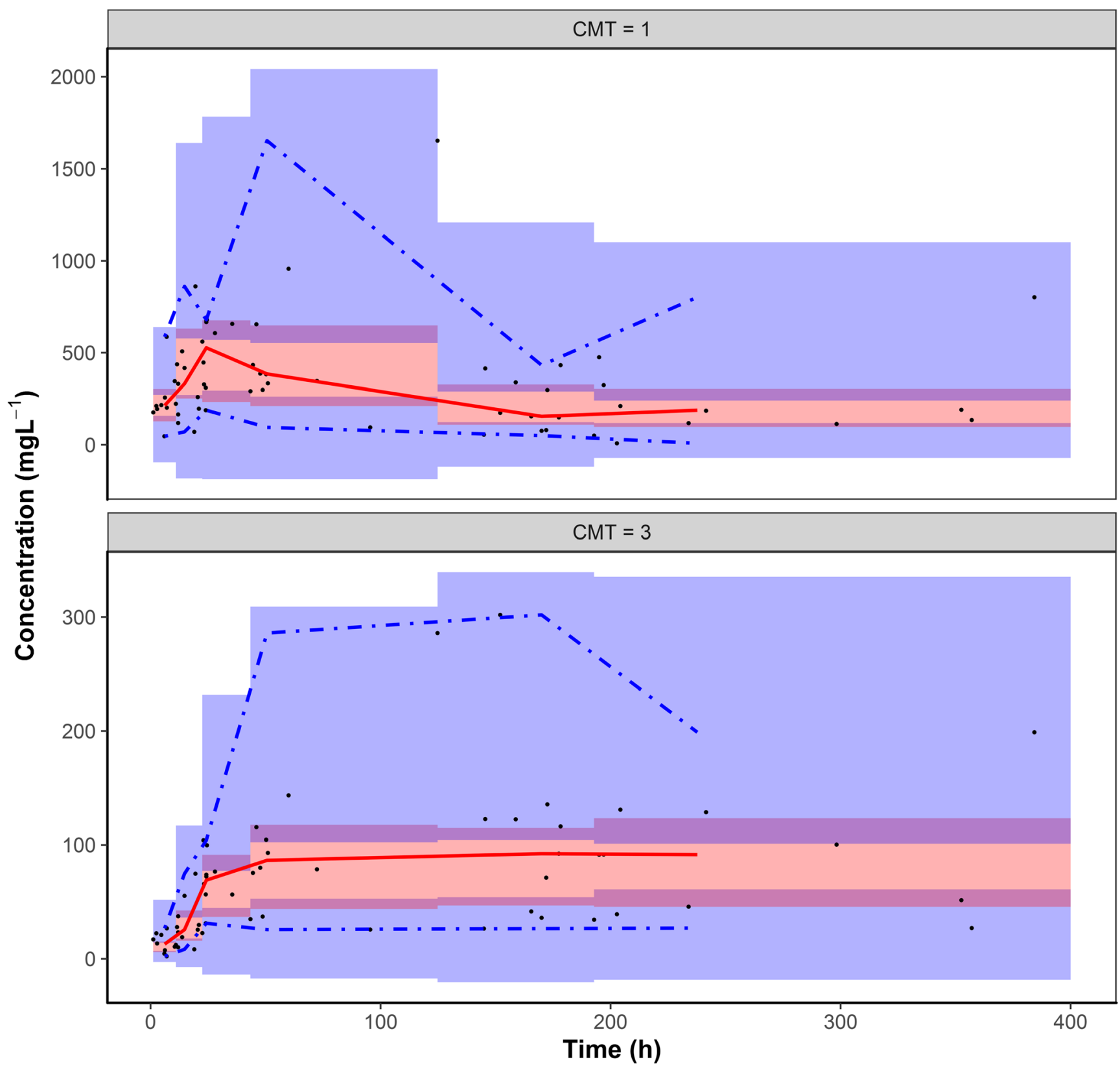

Fig. 3 Visual predictive check of sildenafil (CMT/compartment 1) and DMS (CMT/compartment 3), showing how well the average trend of the observations (solid line) and how well the variability of the observed data (two dashed lines) fall within the model-simulated average trend (red

shaded area) and the model-simulated variability (blue shaded areas) represented as a 95\% confidence interval (CI). The average and the variability of the observed data both fall within the corresponding simulations

We were not able to retain a significant effect of ECMO on our model. However, considering the increase in circulating volume and the use of an oxygenator, one would assume ECMO to be of substantial influence $[37,38]$. We probably did not find this due to the relatively few samples taken in only 23 patients. We observed both lowered sildenafil levels as well as increased levels when on ECMO. Ahsman et al. evaluated the PK of oral sildenafil in infants on ECMO and post-ECMO and also found contradicting results on the influence of ECMO [29, 39]. In comparison to the work of Ahsman, new

ECMO systems are used with smaller tubes and filters. We expect that the effect of ECMO can be better evaluated as part of the external validation performed in the CoDiNOS trial (NTR6982). Bosentan is known to decrease sildenafil plasma concentrations, but it was not a significant covariate in our study because only one patient was co-treated with bosentan [40]. Sildenafil loading dose and continuous infusion seem to be tolerated well as in only two patients sildenafil had to be temporarily decreased or interrupted due to hypotension. However, because of the retrospective character of the study, 
further analysis of the cardiovascular tolerance of sildenafil in this patient group, using VIS, was not possible. Kipfmueller et al. found an acute improvement of OI but also the needs to increase inotropic support in patients with $\mathrm{CDH}$ [30]. In a prospective setting, these secondary outcome parameters can be evaluated much more reliable.

This is the first study on the PK of intravenous sildenafil in a representative population of $\mathrm{CDH}$ patients in need for $\mathrm{PH}$ treatment. Using population PK modeling, a sildenafil plasma concentration model was developed with sparse sampling. In conclusion, intravenous sildenafil loading dose of $0.4 \mathrm{mg} / \mathrm{kg}$ for $3 \mathrm{~h}$ followed by continuous infusion of $1.6 \mathrm{mg} / \mathrm{kg} / \mathrm{day}$ achieves adequate sildenafil plasma levels. Only postnatal age influences its clearance. This dosing regimen was well tolerated in this small group of $\mathrm{CDH}$ patients. The current PK model is the first step toward concentration targeted sildenafil dosing in $\mathrm{CDH}$ patients. The model will be further validated in the CoDiNOS trial (NTR6982).

Authors' contributions S.C.d.O and B.K. wrote the article; S.C.d.O., F.K., K.A, and D.T designed the research. S.C.d.O. and F.K performed the research, B.K and B.d.W. developed the model. S.C.d.O., F.K., K.A, D.T., A.M., and B.K analyzed the data.

\section{Compliance with ethical standards}

Conflict of interest The authors declare that they have no conflict of interest.

Open Access This article is distributed under the terms of the Creative Commons Attribution 4.0 International License (http:// creativecommons.org/licenses/by/4.0/), which permits unrestricted use, distribution, and reproduction in any medium, provided you give appropriate credit to the original author(s) and the source, provide a link to the Creative Commons license, and indicate if changes were made.

\section{References}

1. Robinson PD, Fitzgerald DA (2007) Congenital diaphragmatic hernia. Paediatr Respir Rev 8(4):323-334 quiz 334-5

2. Snoek KG et al (2016) Standardized postnatal management of infants with congenital diaphragmatic hernia in Europe: the $\mathrm{CDH}$ EURO consortium consensus - 2015 Update. Neonatology 110(1):66-74

3. Reiss I et al (2010) Standardized postnatal management of infants with congenital diaphragmatic hernia in Europe: the CDH EURO consortium consensus. Neonatology 98(4):354-364

4. Snoek KG et al (2016) Conventional mechanical ventilation versus high-frequency oscillatory ventilation for congenital diaphragmatic hernia: a randomized clinical trial (the VICI-trial). Ann Surg 263(5):867-874

5. Logan JW et al (2007) Mechanical ventilation strategies in the management of congenital diaphragmatic hernia. Semin Pediatr Surg 16(2):115-125

6. Lally KP (2002) Congenital diaphragmatic hernia. Curr Opin Pediatr 14(4):486-490
7. Vali P, Lakshminrusimha S (2017) The fetus can teach us: oxygen and the pulmonary vasculature. Children (Basel) 4(8)

8. Kool $\mathrm{H}$ et al (2014) Pulmonary vascular development goes awry in congenital lung abnormalities. Birth Defects Res C Embryo Today 102(4):343-358

9. Noori S et al (2007) Cardiovascular effects of sildenafil in neonates and infants with congenital diaphragmatic hernia and pulmonary hypertension. Neonatology 91(2):92-100

10. Bialkowski A, Moenkemeyer F, Patel N (2015) Intravenous sildenafil in the management of pulmonary hypertension associated with congenital diaphragmatic hernia. Eur J Pediatr Surg 25(2):171-176

11. Kipfmueller $\mathrm{F}$ et al (2018) Continuous intravenous sildenafil as an early treatment in neonates with congenital diaphragmatic hernia. Pediatr Pulmonol 53(4):452-460

12. Kelly LE, Ohlsson A, Shah PS (2017) Sildenafil for pulmonary hypertension in neonates. Cochrane Database Syst Rev 8: CD005494

13. Mukherjee A et al (2009) Population pharmacokinetics of sildenafil in term neonates: evidence of rapid maturation of metabolic clearance in the early postnatal period. Clin Pharmacol Ther 85(1):5663

14. Cheitlin MD et al (1999) ACC/AHA expert consensus document. Use of sildenafil (viagra) in patients with cardiovascular disease. American College of Cardiology/American Heart Association. J Am Coll Cardiol 33(1):273-282

15. Samiee-Zafarghandy S, Smith PB, van den Anker JN (2014) Safety of sildenafil in infants*. Pediatr Crit Care Med 15(4):362-368

16. Steinhorn RH et al (2009) Intravenous sildenafil in the treatment of neonates with persistent pulmonary hypertension. J Pediatr 155(6): $841-847$ e1

17. Leeuwen L et al (2018) Risk factors of impaired neuropsychologic outcome in school-aged survivors of neonatal critical illness. Crit Care Med 46(3):401-410

18. Guidance of industry bioanalytical method validation 2001

19. Karlsson MO, Savic RM (2007) Diagnosing model diagnostics. Clin Pharmacol Ther 82(1):17-20

20. Sharma V, McNeill JH (2009) To scale or not to scale: the principles of dose extrapolation. Br J Pharmacol 157(6):907-921

21. Ette EI (1997) Stability and performance of a population pharmacokinetic model. J Clin Pharmacol 37(6):486-495

22. Bergstrand $\mathrm{M}$ et al (2011) Prediction-corrected visual predictive checks for diagnosing nonlinear mixed-effects models. AAPS J 13(2):143-151

23. Comets E, Brendel K, Mentre F (2008) Computing normalised prediction distribution errors to evaluate nonlinear mixed-effect models: the npde add-on package for R. Comput Methods Prog Biomed 90(2):154-166

24. Baquero $\mathrm{H}$ et al (2006) Oral sildenafil in infants with persistent pulmonary hypertension of the newborn: a pilot randomized blinded study. Pediatrics 117(4):1077-1083

25. Uslu S et al (2011) A comparison of magnesium sulphate and sildenafil in the treatment of the newborns with persistent pulmonary hypertension: a randomized controlled trial. J Trop Pediatr 57(4): $245-250$

26. Al Omar S et al (2016) Effect of early adjunctive use of oral sildenafil and inhaled nitric oxide on the outcome of pulmonary hypertension in newborn infants. A feasibility study. J Neonatal-Perinatal Med 9(3):251-259

27. Vargas-Origel A et al (2010) The use of sildenafil in persistent pulmonary hypertension of the newborn. Am J Perinatol 27(3): $225-230$

28. Herrera TR, Concha G, Holberto CJ, Loera GRG, Rodriguez BI (2006) Oral sildenafil as an alternative treatment in the persistent pulmonary hypertension in newborns [sildenafil oral como alternativo en el tratamiento de recien nacidos con hipertension pulmonar persistente]. Rev Mex Pediatr 73(4):159-163 
29. Ahsman MJ et al (2010) Sildenafil exposure in neonates with pulmonary hypertension after administration via a nasogastric tube. Arch Dis Child Fetal Neonatal Ed 95(2):F109-F114

30. Kipfmueller F et al (2018) Continuous intravenous sildenafil as an early treatment in neonates with congenital diaphragmatic hernia. Pediatr Pulmonol

31. Hyland R et al (2001) Identification of the cytochrome P450 enzymes involved in the $\mathrm{N}$-demethylation of sildenafil. Br J Clin Pharmacol 51(3):239-248

32. Kearns GL et al (2003) Developmental pharmacology-drug disposition, action, and therapy in infants and children. N Engl J Med 349(12):1157-1167

33. Ince I et al (2013) A novel maturation function for clearance of the cytochrome P450 3A substrate midazolam from preterm neonates to adults. Clin Pharmacokinet 52(7):555-565

34. Treluyer JM et al (1997) Developmental expression of CYP2C and CYP2C-dependent activities in the human liver: in-vivo/in-vitro correlation and inducibility. Pharmacogenetics 7(6):441-452

35. Abduljalil $\mathrm{K}$ et al (2014) Changes in individual drug-independent system parameters during virtual paediatric pharmacokinetic trials: introducing time-varying physiology into a paediatric PBPK model. AAPS J 16(3):568-576
36. Brussee JM et al (2018) Predicting CYP3A-mediated midazolam metabolism in critically ill neonates, infants, children and adults with inflammation and organ failure. Br J Clin Pharmacol 84(2): 358-368

37. Wildschut ED et al (2010) Determinants of drug absorption in different ECMO circuits. Intensive Care Med 36(12):2109-2116

38. Raffaeli $\mathrm{G}$ et al (2018) In vitro adsorption of analgosedative drugs in new extracorporeal membrane oxygenation circuits. Pediatr Crit Care Med 19(5):e251-e258

39. Ahsman MJ, Wildschut ED, Witjes BCM, Tibboel D, Mathot RAA Population pharmacokinetics of oral sildenafil during and after extracorporeal membrane oxygenation in neonates. in Determinants of pharmacokinetic variability during extracorporeal membrane oxygenation. A roadmap to rational pharmacotherapy in children 2010, Erasmus Medical Center Rotterdam: Rotterdam, The Netherlands p 133-149

40. Burgess G et al (2008) Mutual pharmacokinetic interactions between steady-state bosentan and sildenafil. Eur J Clin Pharmacol 64(1):43-50

Publisher's note Springer Nature remains neutral with regard to jurisdictional claims in published maps and institutional affiliations. 